\title{
THE STRONG CLOSURE OF BOOLEAN ALGEBRAS OF PROJECTIONS IN BANACH SPACES
}

\author{
J. DIESTEL and W. J. RICKER \\ (Received 1 May 2003; revised 16 September 2003) \\ Communicated by A. J. Pryde
}

\begin{abstract}
This note improves two previous results of the second author. They turn out to be special cases of our main theorem which states: A Banach space $X$ has the property that the strong closure of every abstractly $\sigma$-complete Boolean algebra of projections in $X$ is Bade complete if and only if $X$ does not contain a copy of the sequence space $\ell^{\infty}$.
\end{abstract}

2000 Mathematics subject classification: primary 06E15, 46B25, 47L10.

\section{Statement of results}

Let $X$ be a Banach space and $\mathscr{B}$ be a Boolean algebra (briefly, B.a.) of continuous projections in $X$; the partial order is range inclusion, that is, $B_{1} \leq B_{2}$ means $B_{1} X \subseteq$ $B_{2} X$, and the unit is the identity operator $I$ in $X$. Recall that $\mathscr{B}$ is called Bade complete (respectively Bade $\sigma$-complete) if $\mathscr{B}$ is complete (respectively $\sigma$-complete) as an abstract B.a. and, for each family (respectively countable family) $\left\{B_{\alpha}\right\} \subseteq \mathscr{B}$, we have

$$
\left(\vee_{\alpha} B_{\alpha}\right) X=\overline{\operatorname{span}\left\{\bigcup_{\alpha} B_{\alpha} X\right\}} \text { and }\left(\wedge_{\alpha} B_{\alpha}\right) X=\cap_{\alpha} B_{\alpha} X ;
$$

see, for example, [1, Chapter XVII]. The space of all continuous linear operators of $X$ into itself is denoted by $\mathscr{L}(X)$; it is equipped with the strong operator topology. The dual Banach space of $X$ is denoted by $X^{*}$.

The aim of this short note is to extend the two main results of [7]; they both turn out to be special cases of the following single result.

(C) 2004 Australian Mathematical Society 1446-7887/04 \$A2.00+0.00 
THEOREM. A Banach space $X$ has the property that the strong closure (that is, in $\mathscr{L}(X))$ of every abstractly $\sigma$-complete B.a. of projections in $X$ is Bade complete if and only if $X$ does not contain a copy of $\ell^{\infty}$.

Theorem 2 of [7] states that if a Banach space $X$ is weakly compactly generated (briefly, WCG), then the strong closure of any abstractly $\sigma$-complete B.a. of projections in $X$ is Bade complete. It is known that WCG spaces cannot contain a copy of $\ell^{\infty}$, [7, page 283]. Moreover, there exist Banach spaces $X$ which do not contain a copy of $\ell^{\infty}$, but fail to be WCG, [7, Remarks 1 (i) and 3 (i)]. So, the above theorem is a genuine extension of [7, Theorem 2].

Theorem 3 of [7] states that a Banach space $X$ has the property that the strong closure of every abstractly complete B.a. of projections in $X$ is Bade complete if, and only if, $X$ does not contain a copy of $\ell^{\infty}$. Our main theorem is also an extension of this result; it relaxes the requirement of abstract completeness to abstract $\sigma$-completeness. Again the extension is genuine. For instance, let $X:=\ell^{p}([0,1])$ for any $1 \leq p<\infty$ and define $\mathscr{B}:=\{P(E): E$ a Borel subset of $[0,1]\}$ where, for each such Borel set $E$, the projection $P(E) \in \mathscr{L}(X)$ is defined by $P(E) \varphi=\chi_{E} \varphi$ (pointwise product on $[0,1])$ and each $\varphi \in X$ is considered as a $\mathbb{C}$-valued function on $[0,1]$. Then $\mathscr{B}$ is an abstractly $\sigma$-complete B.a. in $\mathscr{L}(X)$ which is not abstractly complete.

Further related results, due to Gillespie, can be found in $[3,2]$.

The extension of the above mentioned results in [7] is possible because of the following fact (answering Question 1 in [7]). Recall that a compact, totally disconnected Hausdorff space $\Omega$ is called $\sigma$-Stonian (or basically disconnected) if the closure of the union of any countable family of clopen sets (that is, simultaneously closed and open) is an open set. The space $C(\Omega)$, consisting of all $\mathbb{C}$-valued continuous functions on $\Omega$, is equipped with the sup-norm.

Proposition A. Let $\Omega$ be a $\sigma$-Stonian space and $X$ be a Banach space not containing a copy of $\ell^{\infty}$. Then every continuous linear operator from $C(\Omega)$ into $X$ is necessarily weakly compact.

Let us accept this result for the moment.

PROOF OF THEOREM. Suppose that $X$ does not contain a copy of $\ell^{\infty}$. A careful examination of the proof of [7, Theorem 2] reveals that it also carries over to the current setting, provided that we now replace the use of [7, Proposition 1] in that proof with Proposition A above.

Conversely, suppose that $X$ does contain a copy of $\ell^{\infty}$. The same example constructed in the proof of [7, Theorem 3] also applies here (since every abstractly complete B.a. is also abstractly $\sigma$-complete) to show that there necessarily exists an 
abstractly $\sigma$-complete, strongly closed B.a. of projections in $X$ which fails to be Bade complete.

REMARK. An abstractly $\sigma$-complete B.a. of projections in a Banach space not containing a copy of $\ell^{\infty}$ need not itself be Bade complete or even Bade $\sigma$-complete, [7, Remark 2].

So, back to Proposition A which is a reformulation of the following result, due to Rosenthal, [8, Theorem 3.7]; see also [6, Theorem 5.3.17 and Corollaries 3.4.5 and 5.3.14] in the setting of Banach lattices. Recall that a continuous linear operator $T: X \rightarrow Y$, with $X$ and $Y$ Banach spaces, is called an isomorphism (of $X$ into $Y$ ) if it is injective and its range $T X$ is a closed subspace of $Y$. We also say that $Y$ contains a copy of $X$.

PROPOSITION B. Let $\Omega$ be a $\sigma$-Stonian space and $X$ be a Banach space. Let $T: C(\Omega) \rightarrow X$ be a continuous linear operator which fails to be weakly compact. Then there exists a closed subspace $X_{0}$ of $C(\Omega)$ which is isometrically isomorphic to $\ell^{\infty}$ and such that the restriction $\left.T\right|_{X_{0}}: X_{0} \rightarrow X$ is an isomorphism of $X_{0}$ into $X$.

The proof of this result given in [8] is not entirely clear, especially the reference made to [4] (of our references) in the proof of [8, Proposition 3.6], which is then used in the proof of the main result, [8, Theorem 3.7]. Since we know of no other reference to Proposition B, for the sake of completeness we include a (perhaps) more transparent and self-contained proof of it. Some preliminaries will be required.

LEMMA 1 ([8, Lemma 1.1 (a)]). Let $\Omega$ be a $\sigma$-Stonian space and $\left\{\mu_{n}\right\}_{n=1}^{\infty}$ be a bounded sequence in $C(\Omega)^{*}$. Suppose that $\left\{E_{n}\right\}_{n=1}^{\infty}$ is a sequence of painwise disjoint clopen subsets of $\Omega$ and let $\varepsilon>0$ be given. Then there exists an infinite subset $M \subseteq \mathbb{N}$ such that

$$
\left|\mu_{m}\right|\left(\overline{\bigcup_{k \neq m} E_{k}}\right)<\varepsilon, \quad m \in M .
$$

Another ingredient needed for the proof of Proposition B is the following result of Grothendieck.

LEMMA 2 ([5, Theoreme 2, page 146]). Let $\Omega$ be a compact Hausdorff space and $K \subseteq C(S)^{*}$ be a bounded set which is not relatively weakly compact. Then there exists $\delta>0$, a sequence $\left\{\mu_{n}\right\}_{n=1}^{\infty} \subseteq K$ and a sequence $\left\{O_{n}\right\}_{n=1}^{\infty}$ of pairwise disjoint open subsets of $\Omega$ such that $\left|\mu_{n}\right|\left(O_{n}\right)>\delta$, for all $n \in \mathbb{N}$.

We now formulate the main fact needed for proving Proposition $B$; it is the $\sigma-$ Stonian version of [8, Proposition 3.6], with 'another proof'. 
Lemma 3. Let $\Omega$ be a $\sigma$-Stonian space, $X$ be a Banach space, $T: C(\Omega) \rightarrow X$ be a continuous linear operator, and $0<\varepsilon<\delta$ be given. Suppose that there exists a sequence $\left\{x_{n}^{*}\right\}_{n=1}^{\infty}$ in the closed unit ball of $X^{*}$ and a sequence $\left\{O_{n}\right\}_{n=1}^{\infty}$ of pairwise disjoint open subsets of $\Omega$ such that

$$
\left|x_{n}^{*} T\right|\left(O_{n}\right)>\delta \text { and }\left|x_{n}^{*} T\right|\left(\overline{\bigcup_{k \neq n} O_{k}}\right)<\varepsilon,
$$

for every $n \in \mathbb{N}$. Then there exists a closed subspace $X_{0}$ of $C(\Omega)$ such that $X_{0}$ is isometrically isomorphic to $\ell^{\infty}$ and the restriction $\left.T\right|_{X_{0}}$ is an isomorphism.

PROOF. Let $\mu_{n}:=\left|x_{n}^{*} T\right|$, for $n \in \mathbb{N}$, where $x_{n}^{*} T$ denotes the measure representing the element $x_{n}^{*} \circ T$ of $C(\Omega)^{*}$. Using the regularity of $\mu_{n}$ and a compactness argument, we can find a clopen set $P_{n} \subseteq O_{n}$ such that $\mu_{n}\left(P_{n}\right)>\delta$, in which case also $\mu_{n}\left(\overline{\bigcup_{k \neq n} P_{k}}\right)<\varepsilon$. So, we can (and do) assume that each set $O_{n}$, for $n \in \mathbb{N}$, in the statement of the lemma is actually clopen.

Let $U:=\bigcup_{n=1}^{\infty} O_{n}$ and put $F:=\bar{U}$. Then $F$ is clopen in $\Omega$ and $F$ is itself $\sigma$-Stonian (for the relative topology). Actually, $F \simeq \beta(U)$ is the Čech-Stone compactification of the locally compact space $U$. To see this, let $f: U \rightarrow \mathbb{R}$ be any bounded continuous function. For any finite set $A \subseteq \mathbb{N}$, the function $f_{A}:=f \chi_{O(A)}$ belongs to $C(\Omega)$, where $O(A):=\bigcup_{n \in A} O_{n}$. There are countably many such functions $f_{A}$ and, since $\Omega$ is $\sigma$-Stonian, the lattice supremum $g:=\vee_{A} f_{A} \in C(\Omega)$ exists, [4, page 52]. Clearly, $f=\left.g\right|_{U}$.

Choose any $\delta^{\prime} \in(\varepsilon, \delta)$. For each $n \in \mathbb{N}$, choose $\varphi_{n} \in C(\Omega)$ with support in $O_{n}$ and satisfying $\left\|\varphi_{n}\right\|_{\infty}=1$ and $\int_{O_{n}} \varphi_{n} d \mu_{n} \geq \delta^{\prime}$.

Let $X_{0}$ be the collection of all elements $f \in C(\Omega)$ such that, on $O_{n}$, the function $f$ is a constant multiple of $\varphi_{n}$, for each $n \in \mathbb{N}$. Since $F \simeq \beta(U)$ and, for each $f \in X_{0}$ each restriction $\left.f\right|_{o_{n}}$ is a constant multiple of $\varphi_{n}$ (for every $n \in \mathbb{N}$ ), it is clear that $X_{0}$ is isometrically isomorphic to $\ell^{\infty}$. In particular, $X_{0}$ is a closed subspace of $C(\Omega)$.

To show that $\left.T\right|_{X_{0}}$ is an isomorphism, let $f \in X_{0}$ and $n \in \mathbb{N}$ be fixed. Noting that $F$ is the disjoint union of $O_{n}$ and $\overline{U \backslash O_{n}}$, we have

$$
\begin{aligned}
\left|\left(x_{n}^{*} T\right)(f)\right| & =\left|\int_{F} f d \mu_{n}\right|=\left|\int_{O_{n}} f d \mu_{n}+\int_{\overline{U \backslash O_{n}}} f d \mu_{n}\right| \\
& \geq\left|\int_{O_{n}} f d \mu_{n}\right|-\left|\int_{\overline{U \backslash O_{n}}} f d \mu_{n}\right| \geq \delta^{\prime}\left\|\left.f\right|_{O_{n}}\right\|_{\infty}-\varepsilon\|f\|_{\infty} .
\end{aligned}
$$

Since $\|f\|_{\infty}=\sup _{n}\left\|\left.f\right|_{o_{n}}\right\|_{\infty}$ we conclude that

$$
\|T f\| \geq \sup _{n}\left|\left(x_{n}^{*} T\right)(f)\right| \geq \sup _{n}\left(\delta^{\prime}\left\|\left.f\right|_{o_{n}}\right\|_{\infty}-\varepsilon\|f\|_{\infty}\right)=\left(\delta^{\prime}-\varepsilon\right)\|f\|_{\infty} .
$$


This is valid for every $f \in X_{0}$, from which it follows that $\left.T\right|_{X_{0}}$ is injective and has closed range.

Proof OF Proposition B. Since $T$ is not weakly compact, Lemma 2 ensures the existence of a sequence $\left\{x_{n}^{*}\right\}_{n=1}^{\infty}$ in the closed unit ball of $X^{*}$, a $\delta>0$ and a sequence $\left\{O_{n}\right\}_{n=1}^{\infty}$ of pairwise disjoint open sets in $\Omega$ so that, with $\mu_{n}:=\left|x_{n}^{*} T\right|$, we have $\mu_{n}\left(O_{n}\right)>\delta$ for each $n \in \mathbb{N}$. Arguing as in the proof of Lemma 3, the $\sigma$-Stonian nature of $\Omega$ lets us assume that each $O_{n}$, for $n \in \mathbb{N}$, is actually clopen. By Lemma 1 there is an infinite subset $M \subseteq \mathbb{N}$ so that $\mu_{m}\left(\overline{\bigcup_{k \neq m} O_{k}}\right)<\delta / 2$ for each $m \in M$ and, of course, also $\mu_{m}\left(O_{m}\right)>\delta$ for each $m \in M$. Put $\varepsilon:=\delta / 2$. Then Lemma 3 gives the desired conclusion.

\section{References}

[1] N. Dunford and J. T. Schwartz, Linear operators III: spectral operators (Wiley-Interscience, New York, 1971).

[2] T. A. Gillespie, 'Spectral measures on spaces not containing $\ell^{\infty}$ ', Proc. Edinburgh Math. Soc. (Ser. II) 24 (1981), 41-45.

[3] — 'Strongly closed bounded Boolean algebras of projections', Glasgow Math. J. 22 (1981), 73-75.

[4] L. Gillman and M. Jerison, Rings of continuous functions (van Nostrand, Princeton, 1960).

[5] A. Grothendieck, 'Sur les applications lineaires faiblement compactes d'espaces du type $C(K)$ ', Canad. J. Math. 5 (1953), 129-173.

[6] P. Meyer-Nieberg, Banach lattices (Springer, Berlin, 1991).

[7] W. J. Ricker, 'The strong closure of $\sigma$-complete Boolen algebras of projections', Archiv Math. (Basel) 72 (1999), 282-288.

[8] H. P. Rosenthal, 'On relatively disjoint families of measures with some applications to Banach space theory', Studia Math. 37 (1970), 13-36.

Department of Mathematical Sciences

Kent State University

P.O. Box 5190

Kent OH 44242-0001

USA

e-mail: diestelj@aol.com
Math.-Geogr. Fakultät

Katholische Universität

Eichstätt-Ingolstadt

D-85072 Eichstätt

Germany

e-mail: werner.ricker@ku-eichstaett.de 
